\title{
Slotted ALOHA and Code Division Multiple Access Techniques for Land-Mobile Satellite Personal Communications
}

Richard D. J. van Nee, Rogier N. van Wolfswinkel, Student Member, IEEE, and Ramjee Prasad, Senior Member, IEEE

\begin{abstract}
The throughput and delay characteristics of a landmobile satellite channel are analyzed for both slotted ALOHA and slotted direct-sequence CDMA (code division multiple access), using binary phase shift keying (BPSK) modulation and forward error correction coding (FEC). In the case of CDMA, the application of path diversity techniques-maximal ratio combining and selection diversity-is also taken into account. Packet success probabilities are derived for both slow and fast fading, in order to evaluate the throughput and delay. Numerical results are presented for arbitrary code lengths and for specific values of the number of resolvable paths. It is shown that CDMA can offer a substantial improvement over slotted ALOHA, especially when the chip time is less than the delay spread.
\end{abstract}

\section{INTRODUCTION}

$\mathbf{U}$ NIVERSAL personal communication system (UPCS) is defined as the ultimate goal of today's communications engineers, which will be achieved by providing communication services by any person to any person at any place at any time without any delay in any form through any medium using one pocketized unit. In order to achieve this objective, it is necessary to combine the research activities of macro-, micro- and pico-cellular radio systems with the research in the area of satellite communication systems. A number of studies and developments have been carried out for land-mobile satellite communication systems to supplement terrestrial cellular communication systems [1]-[6]. One of the important issues which has to be investigated thoroughly for mobile satellite communication systems is the multiple access technique.

For some years now, a debate is going on whether narrowband or spread-spectrum techniques should be used in order to achieve a high channel capacity. In [4] and [6], it is concluded that code division multiple access (CDMA) yields a better performance than narrowband frequency division multiple access (FDMA) in the case of circuit switched mobile satellite communications. Therefore, CDMA is a strong candidate for the choice of multiple access techniques for future systems. In [7]-[13], it is pointed out that CDMA can also be beneficial in the case of packet-switched radio networks. However, the analysis of multiple access techniques in [7]-[10] did not take into account the fading characteristics of the radio channel,

Manuscript received January 15, 1994; revised July 30, 1994.

The authors are with the Telecommunications and Traffic-Control Systems Group, Delft University of Technology, 2600 GA Delft, The Netherlands.

IEEE Log Number 9407515. which is usually an important limiting factor, especially for satellite communications.

The influence of the channel characteristics on the performance of slotted CDMA and conventional narrowband slotted ALOHA is the subject of the paper. A generalized land-mobile satellite system, modeled by shadowed Rician fading characteristics [14]-[16], is considered in this paper to investigate the throughput and delay performance of slotted ALOHA and direct-sequence CDMA techniques, using BPSK modulation with error correction and diversity techniques. In section II, the equations for throughput and delay of slotted CDMA and narrowband slotted ALOHA are presented. A description of the radio channel and the resulting expressions for the packet success probability are given in section III. Numerical results are discussed in section IV. Finally, section $\mathrm{V}$ contains the main conclusions.

\section{SLOTTED CDMA vERSUS \\ NARROWBAND SLOTTED ALOHA}

We consider a communication network with sufficient bandwidth to accomodate $N$ users in a TDMA or FDMA (time/frequency division multiple access) system, each transmitting at a bit rate of $1 / T_{b}$ with $T_{b}$ as the bit duration. Instead of a fixed assignment scheme like TDMA and FDMA, now consider a random access slotted CDMA scheme, where the data sequence is spread by a certain spreading code, consisting of $N$ chips per bit. The code length and the total number of codes may be fixed to $N$, but it may also be larger than $N$. The only assumption about the codes that it made in this paper is that they can be approximated by random sequences. Further, it is assumed that the total number of users is large enough to get a Poisson distribution function for the offered traffic. Therefore, the probability $P_{\operatorname{tr}}(k)$ that $k$ packets are generated during a certain times slot is given by

$$
P_{\mathrm{tr}}(k)=\frac{G^{k}}{k !} \exp (-G) \text {. }
$$

Here, $G$ is the average number of transmitted packets per time slot.

When a packet is transmitted, there is a certain success probability $P_{s}(k)$ that it is received correctly. It is assumed that an acknowledgment is sent after successful reception of a packet, so after waiting twice the propagation delay, a user knows if its packet is received or not. Although a receiver itself could estimate if a packet was successfully received 
in a single spot system, acknowledgments are mandatory for multispot systems or for systems that require a zero packet loss probability. When the transmitting user does not receive an acknowledgment for a certain packet, it retransmits that packet after a certain random delay. The steady state throughput of this transmission system is defined as the average number of successfully received packets per time slot, and given by

$$
S=\sum_{k=1}^{K_{\max }} k P_{\mathrm{tr}}(k) P_{s}(k) .
$$

Here, $K_{\max }$ is the maximum number of users that can be simultaneously handled by the system, because the number of receivers or available code words is limited.

It may be noted that slotted ALOHA is a special limiting example of slotted CDMA for $K_{\max }=N=1$. In order to make a fair comparison between CDMA and slotted ALOHA, it is desirable to use the same bandwidth for both systems, which gives two options for the slotted ALOHA case: First, the data rate can be chosen equal to the CDMA chip rate. Second, the data rate can be chosen equal to the CDMA data rate, which makes it possible to divide the total bandwidth in $N$ separate ALOHA channels [17]. In this way, a combination of FDMA and ALOHA is made where each user randomly selects a certain frequency band and a certain time slot. The second option can be expected to achieve higher throughput values, because in the first option, the large data rate will cause considerable intersymbol interference. As a result, the bit error probability and hence the throughput for the first option will always be worse than in the multichannel ALOHA system, where the data rate is $N$ times smaller.

Assuming that the total amount of traffic is randomly distributed over $N$ channels, the throughput of the multichannel slotted ALOHA system can simply be calculated as $N$ times the throughput of one narrowband slotted ALOHA channel, with an offered load that is equal to the total offered load divided by $N$. In fact, it is possible to normalize the obtained throughput values for both CDMA and ALOHA by dividing the total system throughput by $N$. In this way, the corresponding throughput per 'channel' is found.

The corresponding average packet delay $D$ is defined as the number of slot times it takes for a packet to be successfully received. Thus it is the average time duration (in slots) between the packet being offered to the transmitter and the packet being successfully received [13], and is given by

$$
D=1.5+T_{d}+\left[\frac{G}{S}-1\right]\left[\frac{N_{\mathrm{AT}}}{2}+1+2 T_{d}\right]
$$

where $G / S-1$ is the average number of retransmission, $N_{\mathrm{AT}} / 2$ is the mean retransmission delay and $T_{d}$ is the propagation delay.

\section{SYSTEM DESCRIPTION}

The system under consideration consists of a satellite hub station and a number of mobile terminals on the earth's surface, like depicted in Fig. 1. The hub station may act like a repeater or it may process the signals on-board. On-board processing has the advantage that the relative amounts of noise, shadowing the multipath are reduced. Further, it may

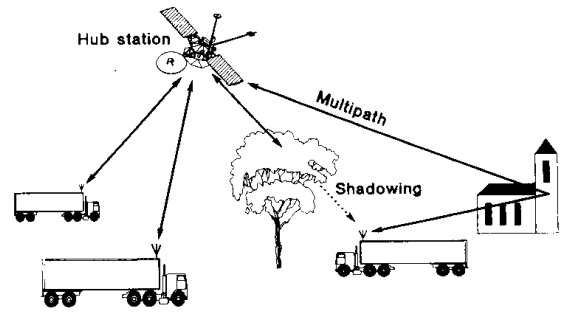

Fig. 1. Land-mobile satellite system.

be necessary to do on-board processing in case of a network of several satellites, or in the case of multiple spot beams per satellite, where the hub station has to make certain routing decision for each message. In this paper, the throughput and delay are analyzed for a single link only.

\section{A. Channel Model}

A statistical propagation model for a narrowband landmobile satellite channel in rural and suburban environments was developed in [14]-[16]. It assumes that the received signal consists of a shadowed line-of-sight signal with a lognormal envelope distribution plus a sum of multipath signals with a Rayleigh distributed envelope. The resulting probability distribution of the received signal envelope $r$ is given by

$$
\begin{aligned}
p_{r}(r)= & \frac{r}{b_{0} \sqrt{2 \pi d_{0}}} \int_{0}^{\infty} \exp \left[-\frac{\left(\ln (z)-\mu_{0}\right)^{2}}{2 d_{0}}-\frac{r^{2}+z^{2}}{2 b_{0}}\right] \\
& \times \frac{I_{0}\left(r z / b_{0}\right)}{z} d z
\end{aligned}
$$

where $I_{0}($.$) is the modified Bessel function of the first kind$ and zeroth order, $b_{0}$ is the average scattered power due to multipath, $\mu_{0}$ is the mean value due to shadowing and $d_{0}$ is the variance due to shadowing.

The narrowband model mentioned above was extended for wideband applications in [18], [19]. In the following, a brief review of this extension is given.

If direct-sequence spread-spectrum modulation is used with a chip duration $T_{c}$ that is less than the delay spread $T_{s}$ of the channel, then the multipath power is partially reduced by the correlation operation in the receiver. Assuming the multipath power delay profile is exponential, the remaining multipath power $b_{m}$ for path $m(m \geq 1)$ can be approximated as

$$
b_{m}=b_{0}\left[1-\exp \left(-\frac{T_{c}}{T_{s}}\right)\right] \exp \left[-(m-1) \frac{T_{c}}{T_{s}}\right] .
$$

In the case of BPSK modulation, the total received signal is

$$
\begin{aligned}
r(t)= & \sum_{k=1}^{K} \sum_{m=1}^{M} A \beta_{m k} a_{k}\left(t-\tau_{m k}\right) b_{k}\left(t-\tau_{m k}\right) \\
& \times \cos \left(\left[\omega_{c}+\omega_{m k}\right] t+\phi_{m k}\right)+n(t)
\end{aligned}
$$

where $m$ and $k$ denote the path and user number, respectively. $A$ is the transmitted signal amplitude, which is assumed to be constant and identical for all users. For user $k,\left\{a_{k}\right\}$ is the spread spectrum code, $\left\{b_{k}\right\}$ is the data sequence, $\omega_{c}+\omega_{m k}$ is the carrier plus Doppler angular frequency, $\phi_{m k}$ is the carried phase, $\tau_{m k}$ is the time delay and $n(t)$ is white Gaussian noise with two-sided spectral density $N_{0} / 2$. The instantaneous path amplitude is denoted as $\beta$. 
The received signal $r(t)$ is converted to baseband and correlated with a particular user code. Assuming that the receiver is able to track the code and carrier phase of path $j$ from user $i$, a signal sample of the correlation output can be written as

$$
z=A \beta_{j i} b_{i}^{0}+\sum_{k=1}^{K} I_{k}+\eta_{i}
$$

where $\eta_{i}$ is a zero-mean Gaussian variable with variance $N_{0} / T_{b}, b_{i}^{0}$ is the current data bit, $T_{b}$ is the bit duration and $I_{k}$ consists of cross correlation products from interfering users and multipath signals. Notice that the equation is slightly different from the one used in [18], [19]. It is now assumed that the correlation operation is normalized by dividing the output by the bit time $T_{b}$. As a result, the equations written in this paper are not dependent on $T_{b}$, contrary to [18], [19]. However, the results are the same, since the signal-to-noise and signal-to-interference ratios are the same in both cases.

In [19], it was shown that a closed form expression can be derived for the variance $\sigma_{i}^{2}$ of the $K$ interfering products. The normalized equation is

$$
\sigma_{i}^{2}=\frac{2 K A^{2}}{3 N}\left(b_{0}+\frac{\exp \left[2 \mu_{0}+2 d_{0}\right]}{2}\right) .
$$

\section{B. Bit Error Probability without Diversity for Narrowband}

The bit error probability for the narrowband BPSK modulation in a shadowed Rician land-mobile satellite channel is given by

$$
P_{e}=\frac{1}{2} \int_{0}^{\infty} \operatorname{erfc}\left(\frac{r}{\sigma_{r} \sqrt{2}}\right) p_{r}(r) d r .
$$

Here, $\sigma_{r}^{2}=N_{0} / T_{b}$ is the noise power, $\operatorname{erfc}(z)$ is the complimentary error function and $p_{r}(r)$ is given by (4).

\section{Bit Error Probability without Diversity for Spread-Spectrum}

If a spread-spectrum receiver only tries to demodulate the first arriving path, containing the line-of-sight signal, then the path gain $\beta_{l i}$ in (7) has a shadowed Rician probability density function. This results in the following bit error probability

$$
P_{e}=\frac{1}{2} \int_{0}^{\infty} \operatorname{erfc}\left(\frac{r}{\sigma \sqrt{2}}\right) P_{\mathrm{rss}}(r) d r .
$$

Here, $\sigma^{2}$ is the sum of the noise and interference power and $\operatorname{erfc}(z)$ is the complimentary error function. For spreadspectrum demodulation the probability density function of the received signal envelope $P_{\text {rss }}(r)$ is obtained by replacing the multipath power $b_{0}$ in (4) by the multipath power $b_{l}$ of the first arriving path, given by.(5)

$$
\begin{aligned}
p_{\text {rss }}(r)= & \frac{r}{b_{1} \sqrt{2 \pi d_{0}}} \int_{0}^{\infty} \exp \left[-\frac{\left(\ln (z)-\mu_{0}\right)^{2}}{2 d_{0}}-\frac{r^{2}+z^{2}}{2 b_{1}}\right] \\
& \times \frac{I_{0}\left(r z / b_{1}\right)}{z} d z .
\end{aligned}
$$

\section{Bit Error Probability with Diversity}

If spread-spectrum modulation is used with a chip time that is less than the delay spread of the channel, then a number of resolvable paths $M$ exist that can be used to improve the performance. When maximal ratio combining is used, the received signal is coherently correlated with a particular code for $M_{d}$ different paths, where $M_{d}$ is the order of diversity. Each path is multiplied by the path gain $\beta_{m k}$ and all correlation outputs are combined. The probability density function of the sum of the squared path gains $\beta_{m k}^{2}$ is the convolution of the $M_{d}$ different squared path gain probability density functions. The resulting probability density function is [19]

$$
\begin{aligned}
p_{\text {mre }}(\alpha)= & \int_{0}^{\alpha} \frac{p_{\text {rss }}(\sqrt{x})}{2 \sqrt{x}} \sum_{i=2}^{M_{d}} \frac{\left(2 b_{i}\right)^{M_{d}-3}}{\prod_{\substack{M_{d} \\
j \neq 2 \\
j \neq i}}^{M_{2}}\left(2 b_{i}-2 b_{j}\right)} \\
& \times \exp \left[-\frac{(x-\alpha)}{2 b_{i}}\right] d x
\end{aligned}
$$

where $\alpha=\sum_{m=1}^{M} \beta_{m k}^{2}$, and $p_{\mathrm{rss}}(\sqrt{x})$ is given by (11)

The bit error probability $P_{e}$ for maximal ratio combining can now be expressed as

$$
P_{e}=\frac{1}{2} \int_{0}^{\infty} \operatorname{erfc}\left[\sqrt{\frac{\alpha A^{2}}{2 \sigma^{2}}}\right] p_{\operatorname{mrc}}(\alpha) d \alpha .
$$

With selection diversity, the strongest out of $M_{d}$ different signals is selected.

The resulting probability density function is [19]

$$
p_{\mathrm{sd}}(x)=\frac{d}{d x}\left(\int_{0}^{x} p_{\mathrm{rss}}(r) \prod_{m=2}^{M_{d}}\left[1-\exp \left(\frac{-x^{2}}{2 b_{1}}\right)\right] d r\right)
$$

where $p_{\mathrm{rss}}(r)$ is given by (11).

The bit error probability $P_{e}$ for selection diversity can now be expressed as

$$
p_{e}=\frac{1}{2} \int_{0}^{\infty} \operatorname{erfc}\left(\frac{r}{\sigma \sqrt{2}}\right) p_{\text {sd }}(r) d r .
$$

\section{E. Packet Success Probability}

Using the previously defined expressions (9), (10), (13), and (15) for the bit error probability, it is now possible to calculate the packet success probability, which is needed to obtain the throughput and delay values of slotted CDMA. The packet success probability $P_{s}$ can be evaluated for slow and fast fading. In the case of slow fading, the path gains are assumed to be constant during one packet time. For fast fading, it is assumed that the path gains are uncorrelated for two consecutive data bits. Further, the use of a forward error correcting code is included by the assumption that $t$ bit errors can be corrected from a total number of $N_{p}$ bits per packet.

The resulting expression for the packet success probability in the case of fast fading is

$$
P_{s}=\sum_{j=0}^{t} P_{e}^{j}\left(1-P_{e}\right)^{N_{p}-j}\left(\begin{array}{c}
N_{p} \\
j
\end{array}\right)
$$

where $P_{e}$ is given by (9) and (10) for the case of narrowband and spread-spectrum modulation, respectively, and $P_{e}$ is given by (15) for selection diversity, and by (13) for the case of maximal ratio combining.

In the case of slow fading, the packet success probability becomes

$$
P_{s}=\int_{0}^{\infty} \sum_{j=0}^{t}\left[P_{e}(x)\right]^{j}\left[1-P_{e}(x)\right]^{N_{p}-j}\left(\begin{array}{c}
N_{p} \\
j
\end{array}\right) p(x) d x
$$


TABLE I

Channel Model Parameters

\begin{tabular}{lccc}
\hline & Light & Average & Heavy \\
\hline $\mathrm{b}_{0}$ & 0.158 & 0.126 & 0.0631 \\
$\mu_{0}$ & 0.115 & -0.115 & -3.91 \\
$\sqrt{d}_{0}$ & 0.115 & 0.161 & 0.806 \\
\hline
\end{tabular}

where for narrowband

$$
P_{e}(x)=\frac{1}{2} \operatorname{erfc}\left[\frac{x A}{\sigma_{r} \sqrt{2}}\right], p(x)=p_{r}(x)
$$

for spread-spectrum with no diversity

$$
P_{e}(x)=\frac{1}{2} \operatorname{erfc}\left[\frac{x A}{\sigma \sqrt{2}}\right], p(x)=p_{\mathrm{rss}}(x)
$$

for spread spectrum with selection diversity

$$
P_{e}(x)=\frac{1}{2} \operatorname{erfc}\left[\frac{x A}{\sigma \sqrt{2}}\right], p(x)=p_{\mathrm{sd}}(x)
$$

for spread spectrum with maximal ratio combining

$$
P_{e}(x)=\frac{1}{2} \operatorname{erfc}\left[\sqrt{\frac{x A^{2}}{2 \sigma^{2}}}\right], p(x)=p_{\mathrm{mrc}}(x) .
$$

Now the throughput and delay can be evaluated for different conditions using (1)-(3) and (16)-(21).

\section{COMputational Results}

The throughput and delay are calculated numerically for several parameters. An interesting remark is that the bit error probability only depends on the ratios $T_{s} / T_{c}$, the instantaneous offered load $N / K$ and $E_{b} / N_{0}=A^{2} T_{b} / 2 N_{0}$. This means that it is not necessary to give results for specific values of the chip time and the code length. As a result, many of the plots like shown in [8], [11], [18], and [19] and in many other papers on CDMA can be generalized, as long as they satisfy the assumptions that both $K$ times $M$ and $N$ are large compared to one.

Table I lists the channel parameters of the shadowed Rician probability distribution function. In [18], it was noticed that the variances of the shadowed Rician probability density function was not normalized to one, which causes bit error probabilities that are too optimistic if the variance exceeds one. This problem is solved now by multiplying the noise power with the total signal power $S=2 b_{0}+\exp \left(2 \mu_{0}+2 d_{0}\right)$.

Figs. 2 and 3 show the normalized throughput $(S / N)$ curves of narrowband slotted ALOHA without and with forward error correction, respectively. The results of Fig. 2 and the following figures were obtained for $T_{s} / T_{c}=6.5, E_{b} / N_{0}=20 \mathrm{~dB}, N_{p}=$ 256 bits per packet and $t=0$ or $t=10$ in the absence or presence of forward error correction coding. The values of $N$ and $K_{\max }$ used were $N=127$ chips per bit and $K_{\max }=$ $2 N$ users. However, as explained earlier, the results are also valid for other values of $N$, as long as $N$ is large compared to one. Fig. 4 shows that the normalized throughput of slotted CDMA without error correction is less than that of slotted ALOHA. However, when error correction is applied, like in the Figs. 3 and 5, then CDMA benefits far more than slotted

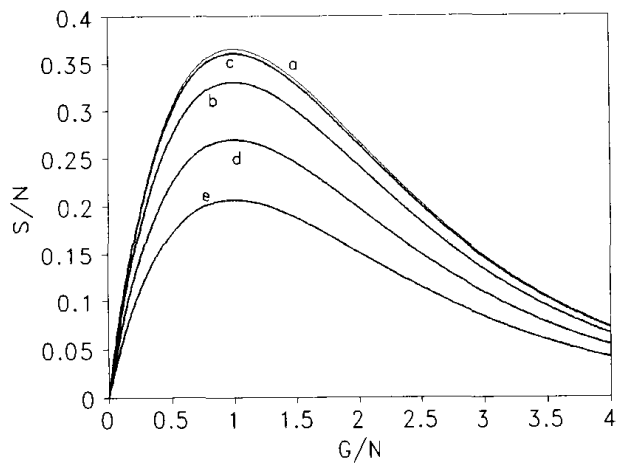

Fig. 2. Normalized throughput of narrowband slotted ALOHA ( $K_{\max }=N=1$ ) for: (a) light shadowing, slow fading; (b) light shadowing, fast fading; (c) average shadowing, slow fading; (d) average shadowing, fast fading; (e) heavy shadowing, slow fading; (f) heavy shadowing, fast fading.

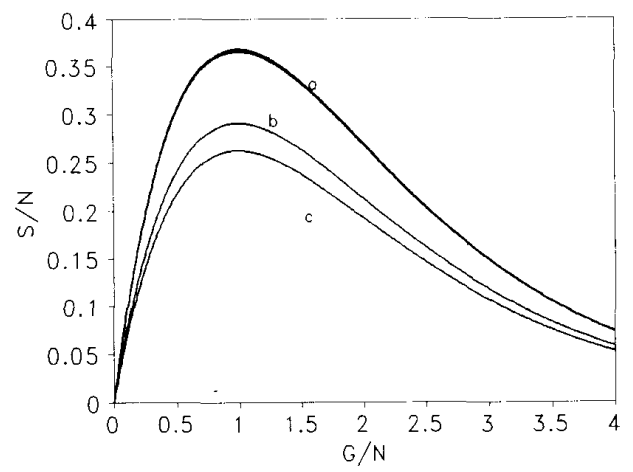

Fig. 3. Normalized throughput of narrowband slotted ALOHA $\left(K_{\max }=N=1\right)$ using FEC coding for: (a) light and average shadowing, slow fading and fast fading; (b) heavy shadowing, slow fading; (c) heavy shadowing, fast fading.

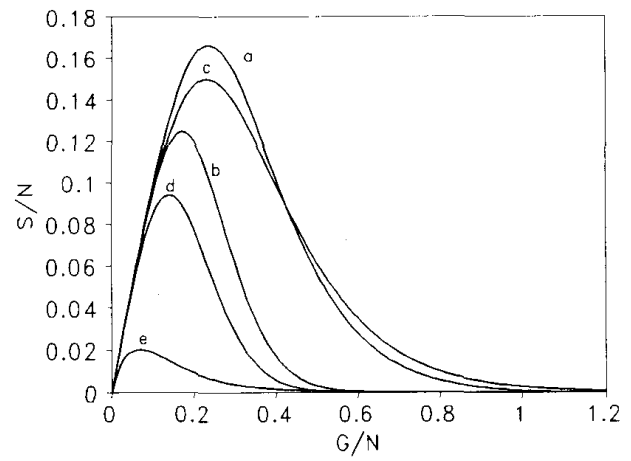

Fig. 4. Normalized throughput of slotted CDMA for: (a) light shadowing, slow fading; (b) light shadowing, fast fading; (c) average shadowing, slow fading; (d) average shadowing, fast fading; (e) heavy shadowing, slow fading; (f) heavy shadowing, fast fading.

ALOHA, which results in a larger normalized throughput than narrowband slotted ALOHA for light and average shadowing.

In Fig. 6, it is demonstrated that the user of maximal ratio combining provides a considerable improvement of the 


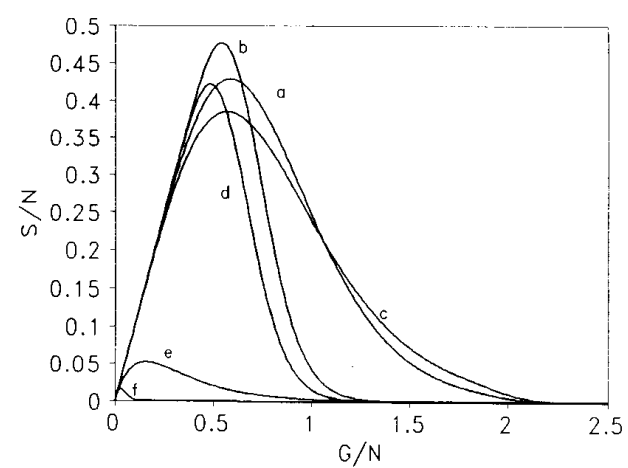

Fig. 5. Normalized throughput of slotted CDMA, using FEC coding for: (a) light shadowing, slow fading; (b) light shadowing, fast fading; (c) average shadowing, slow fading; (d) average shadowing, fast fading; (e) heavy shadowing, slow fading; (f) heavy shadowing, fast fading.

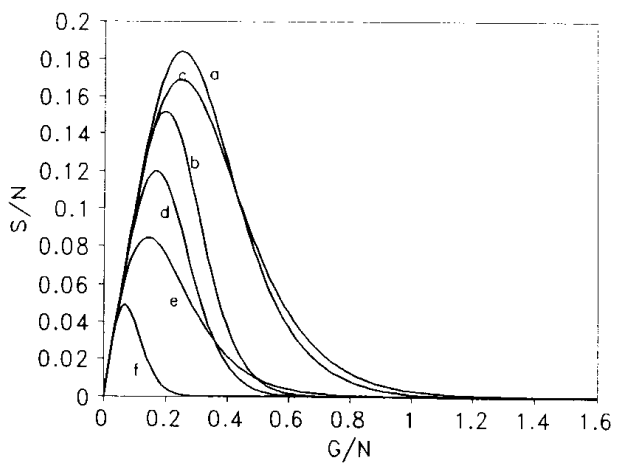

Fig. 6. Normalized throughput of slotted CDMA, using MRC for: (a) light shadowing, slow fading; (b) light shadowing, fast fading; (c) average shadowing, slow fading; (d) average shadowing, fast fading; (e) heavy shadowing, slow fading; (f) heavy shadowing, fast fading.

throughput as compared to Fig. 4. However, if the throughput curves in the case of combined forward error correction and maximal ratio coming (Fig. 7) are compared with the case of just using forward error correction (Fig. 5), then there is only a minor throughput improvement of about ten percent due to maximal ratio combining in the cases of light and average shadowing. For heavy shadowing, however, the use of maximal ratio combining increases the throughput by almost a factor of four. This is because for heavy shadowing, the line-of-slight signal power is negligible as compared to the multipath power. So if the receiver only tries to demodulate the first path, it only uses a small fraction of the total received power, assuming that the chip time is smaller than the multipath delay spread. In that case, maximal ratio combining is very useful to make use of all received signal power that is available.

An interesting fact that can be seen in the previous figures, is that fast fading yields a higher maximum throughput than slow fading when forward error correction coding is applied, while the performance of fast fading is worse if no error correction is used. This is because for fast fading, the bit errors are randomly spread over all packets, so each packet has the same success probability, which is relatively low when no

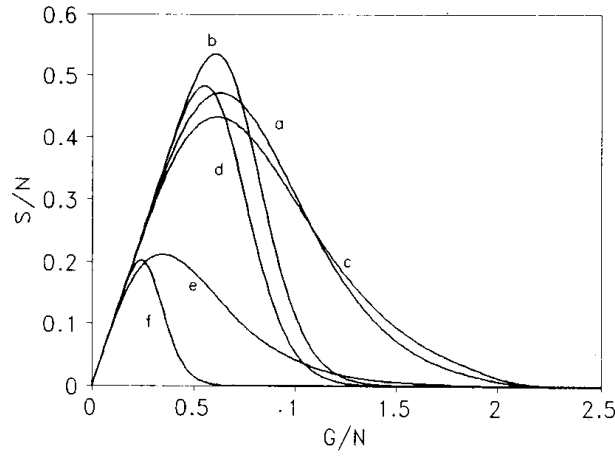

Fig. 7. Normalized throughput of slotted CDMA, using MRC and EFC for: (a) light shadowing, slow fading; (b) light shadowing, fast fading: (c) average shadowing, slow fading; (d) average shadowing, fast fading; (e) heavy shadowing, slow fading; (f) heavy shadowing, fast fading.

error correction coding is used. In the case of slow fading, however, errors appear in bursts. Due to the slow fading, the packet success probability varies, which means that compared to fast fading, there is a certain part of the packets that has a higher packet success probability, resulting in a higher throughput when no forward error correction is applied. If error correction coding is used, then the packet success probability is greatly enhanced for fast fading as long as the signal-to-noise plus interference ratio - which is inversely proportional to the offered load-is above some threshold value. Beneath that threshold, the packet success probability quickly drops to zero, as can be seen in the figures. For slow fading, error correction coding is less effective, because there are less packets with up to $t$ bit errors that can be corrected. Therefore, the maximum throughput in the case of slow fading is less than for fast fading. However, for high values of the offered load, the throughput for slow fading decreases less fast than for fast fading, because there is always a certain part of the packets with a higher success probability than in the case of fast fading.

In Fig. 8, the normalized throughput curves for heavy shadowing using selection diversity are drawn. It is clear that selection diversity performs worse than maximal ratio combining by at least a factor of two. However, it still considerably improves the throughput as compared to the case of no diversity.

Fig. 9 shows the normalized throughput of slotted CDMA with the number of correctable bits as a parameter. As the error correcting capability increases, the maximum achievable throughput increases to high values. However, the user data throughput is of course decreased by the increasing number of bits used for error correction. With $\mathrm{BCH}$ codes, for instance, it is possible to construct error correcting codes with a length of 255 bits and a coding rate close to $2 / 3$ and $1 / 2$, which gives a number of correctable errors $t=11$ or 21 , respectively [20]. These numbers, that are close to the values used in Fig. 9, mean that the maximum net throughput of Fig. 9 is limited to about 0.4

Fig. 10 demonstrates the influence of the ratio $T_{s} / T_{c}$ on the performance of CDMA. It can be seen that the best performance is obtained for a high $T_{s} / T_{c}$ ratio. When $T_{s} / T_{c}$ 


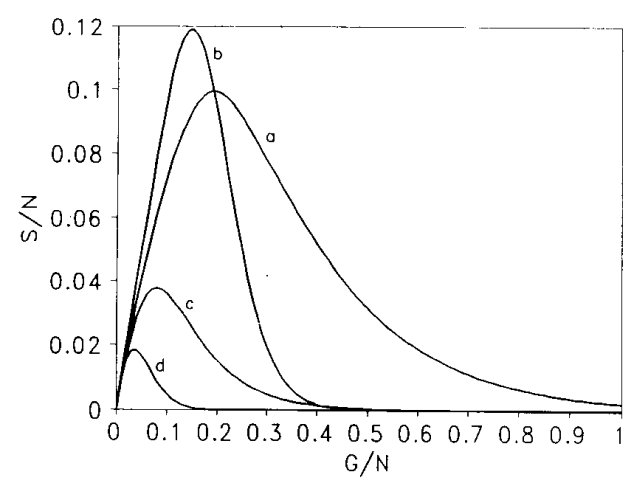

Fig. 8. Normalized throughput of slotted CDMA for heavy shadowing, using selection diversity: (a) FEC, slow fading; (b) FEC, fast fading; (c) no FEC, slow fading; (d) no FEC, fast fading.

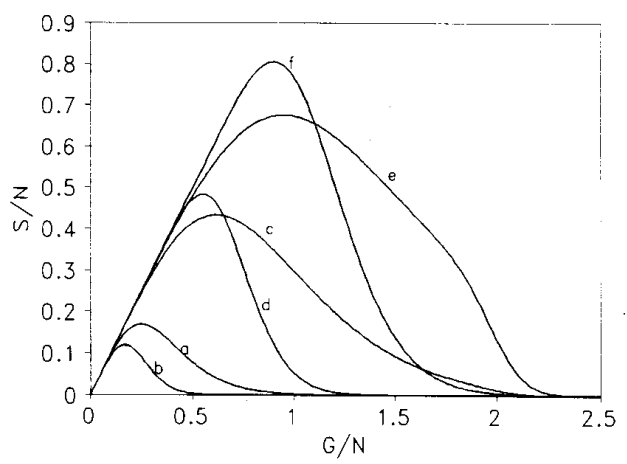

Fig. 9. Normalized throughput of slotted CDMA for average shadowing, using MRC and FEC, with the number of correctable bits as a parameter: (a) $t=0$, slow fading; (b) $t=0$, fast fading; (c) $t=10$, slow fading; (d) $t=$ 10, fast fading; (e) $t=20$, slow fading; (f) $t=20$, fast fading.

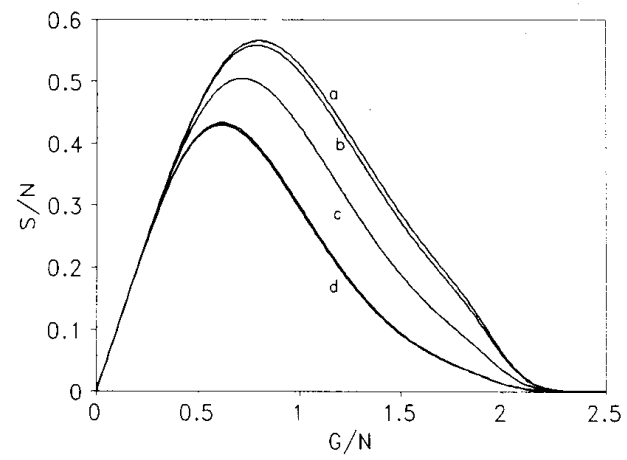

Fig. 10. Normalized throughput of slotted CDMA for average shadowing, slow fading, using MRC and FEC, with $T_{s} / T_{c}$ as a parameter: (a) $T_{s} / T_{c}=10^{4}$; (b) $T_{s} / T_{c}=10^{3}$; (c) $T_{s} / T_{c}=10^{2}$; (d) $T_{s} / T_{c}=10$ and 1 .

is in the order of one or less, then there is practically no benefit anymore of using spread-spectrum to reduce multipath interference. As a result, the performance converges to a certain lower bound. When $T_{s} / T_{c}$ is increased, then the performance increases up to a certain upper bounds, where the multipath interference in the first path, containing the line-of-sight signal, becomes negligible.

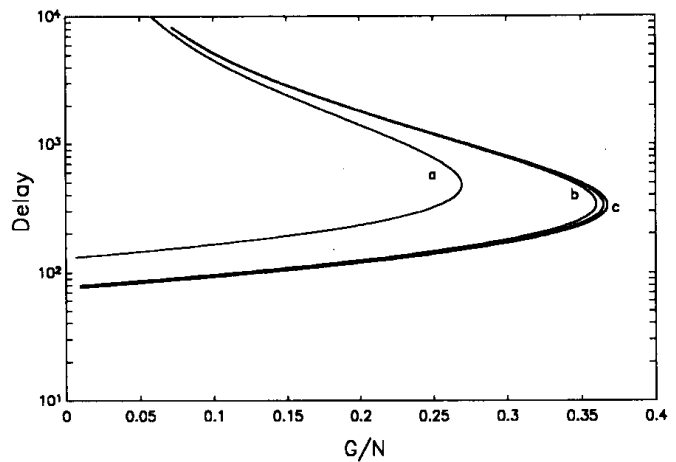

Fig. 11. Normalized throughput-delay curves of narrowband slotted ALOHA for average shadowing: (a) slow fading, no FEC; (b) fast fading, no FEC; (c) slow and fast fading, FEC.

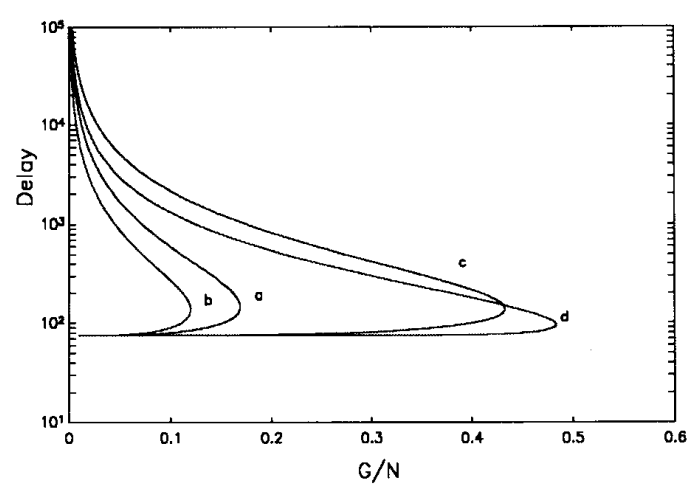

Fig. 12. Normalized throughput-delay curves of slotted CDMA for average shadowing, using MRC: (a) slow fading, no FEC; (b) fast fading, no FEC; (c) slow fading, FEC; (d) fast fading, FEC.

Figs. 11 and 12 show the delay versus the normalized throughput. The results were obtained for values of $N_{\mathrm{at}}=$ 3 and $T_{d}=74$ slots. It can be seen that for narrowband, the difference between slow and fast fading is considerable without error correction, while it becomes negligible when forward error correction is used. In the case of CDMA, fast fading with forward error correction clearly provides the best throughput and delay performance.

\section{CONCLUSION}

The performance of slotted ALOHA and direct sequence CDMA is evaluated for the land-mobile satellite channel in terms of throughput and delay with BPSK modulation for light, average and heavy shadowing. To evaluate the throughput and delay, the packet success probability is derived for narrowband ALOHA with FEC and for spread spectrum with FEC and two types of path diversity (selection diversity and maximal ratio combining), considering slow and fast fading.

It is shown that the performance of CDMA with and without diversity techniques can be generally expressed as a function of $T_{s} / T_{c}, N / K, E_{b} / N_{0}$ and the error correcting capability. Numerical results for the throughout and delay were presented for various combinations of the mentioned parameters. 
Without forward error correction coding, narrowband slotted ALOHA is found to give a better performance than CDMA. However, if error correction coding is applied, CDMA clearly outperforms narrowband slotted ALOHA. The use of forward error correction is far more beneficial for CDMA than the use of path diversity techniques. Maximal ratio combining is very beneficial in the case of heavy shadowing. Slow fading performs better than fast fading, however with FEC fast fading has a higher maximum throughput than slow fading, except for heavy shadowing, where the line-of-sight power is smaller than the multipath power. Further, the throughput of slow fading does not decrease as fast for higher values of the offered load than it does in the case of fast fading.

\section{REFERENCES}

[1] J. H. Lodge, "Mobile satellite communication systems: towards global personal communications," IEEE Commun. Mag., vol. 29, pp. 24-30, Nov. 1991.

[2] P. Wood, "Mobile satellite services for travellers," IEEE Commun. Mag., vol. 29, pp. 32-35, Nov. 1991.

[3] J. L. Grubb, "The traveller's dream come true," IEEE Commun. Mag., vol. 29 , pp. $48-51$, Nov. 1991.

[4] R. J. Leopold, A. Miller, and J. L. Grubb, "The Iridium system: A new paradigm in personal communications," Appl. Microwaves \&Wireless, pp. 68-78, Fall 1993.

[5] K. S. Gilhousen, I. M. Jacobs, R. Padovani, and L. A. Weaver, Jr., "Increased capacity using CDMA for mobile satellite communication," IEEE J. Select., Areas Commun., vol. 8, pp. 503-514, May 1990.

[6] K. G. Johannsen, "Code division multiple access versus frequency division multiple access channel capacity in mobile satellite communication," IEEE Trans. Veh. Technol., vol. 39, pp. 17-26, Feb. 1990.

[7] M. B. Pursley, "The role of spread-spectrum in packet radio networks," Proc. IEEE, vol. 75, Jan. 1987, pp. 116-134.

[8] D. Makrakis and K. M. Sundara Murthy, "Spread slotted ALOHA techniques for mobile and personal satellite communication systems," IEEE J. Select. Areas Commun., vol. 10, pp. 985-1002, Aug. 1992.

[9] D. Raychaudhuri, "Performance analysis of random access packetswitched code division multiple access systems," IEEE Trans. Commun. vol. COM-29, pp. 895-901, June 1981.

[10] K. Joseph and D. Raychaudhuri, "Throughput of unslotted directsequence spread-spectrum multiple-access channels with block FEC coding," IEEE Trans. Commun., vol. 41, pp. 1373-1378, Sept. 1993.

[11] A. Bottcher and M. Dippold, "The capture effect in multi-access communications: The Rayleigh and land-mobile satellite channels," IEEE Trans. Commun., vol. 41, pp. 1364-1372, Sep. 1993.

[12] R. Prasad, C. A. F. J. Wijfels, and K. L. A. Sastry, "Performance analysis of slotted CDMA with DPSK modulation diversity and BCH-coding in indoor radio channels," AEU, Int. J. Elec., Commun., vol. 46, no. 6, pp. $375-382,1992$

[13] C. A. F. J. Wijfels, H. S. Misser, and R. Prasad, "A micro-cellular CDMA system over slow and fast Rician fading radio channels with forward error correcting coding and diversity," IEEE Trans. Veh. Technol., vol, 42, pp. 570-580, Nov. 1993.

[14] C. Loo, "A statistical model for a land-mobile satellite link," IEEE Trans. Veh. Technol., vol VT-34, pp. 122-127, Aug. 1985

[15] - "Measurements and models for a land-mobile satellite channel and their applications to MSK signals," IEEE Trans. Veh. Technol., vol. VT-36, pp. 114-121, Aug. 1987.

[16] _ "Digital transmission through a land-mobile satellite channel," IEEE Trans. Commun., vol. 38, pp. 693-697, May 1990.

[17] W. Yue, "The effect of capture on performance of multichannel slotted ALOHA systems," IEEE Trans. Commun., vol. 39, pp. 818-822, June 1991

[18] R. D. J. van Nee, H. S. Misser, and R. Prasad, "Direct-sequence spreadspectrum in a shadowed Rician fading land-mobile satellite channel," IEEE J. Select. Areas Commun., vol. 10, pp. 350-357, Feb. 1992

[19] R. D. J. van Nee and R. Prasad, "Spread-spectrum path diversity in a shadowed Rician fading land-mobile satellite channel," IEEE Veh. Technol., vol. 42, pp. 131-135, May 1993.

[20] Shu Lin and Daniel Costello, Jr., Error Control Coding: Fundamentals and Applications. Englewood Cliffs, NJ: Prentice-Hall, 1983.

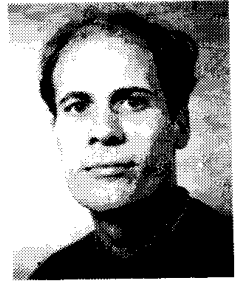

Richard D. J. van Nee was born in Schoonoord, The Netherlands, on January 17, 1967. He received the M.Sc. degree in electrical engineering (cum laude) from the University of Twente, The Netherlands, in 1990. He is working towards the $\mathrm{Ph} . \mathrm{D}$. degree at Delft University of Technology, The Netherlands, where he studies the effects of multipath and multiuser interference on satellite communication and navigation systems.

Mr. van Nee received a best paper award at both the Institute of Navigation GPS-91 conference in Albuquerque, and the Second Annual Conference on Differential Satellite Navigation Systems in Amsterdam, 1993, for his work on the analysis of multipath tracking errors, and the design of a new spread-spectrum receiver architecture which is much less sensitive to multipath than conventional receivers.

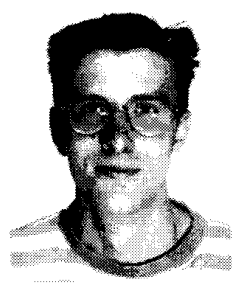

Rogier N. van Wolfswinkel (S'94) was born in The Hague, The Netherlands, on April 1, 1972. He is working toward his M.Sc. degree in electrical engineering.

He is currently with the Telecommunications and Traffic-Control Systems Group at Delft University of Technology (DUT), The Netherlands, His research interests are in the areas of spread-spectrum and satellite communications.

Mr. van Wolfswinkel is the secretary of the Avionics students' society, at DUT.

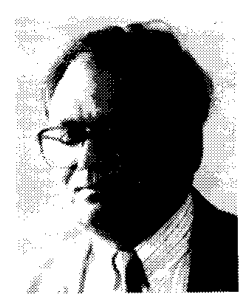

Ramjee Prasad (M'88-SM'90) was born in Babhnaur (Gaya), Bihar, India, on July 1, 1946. He received the B.Sc. (Eng.), M.Sc. (Eng.), and the Ph.D. degrees from Birla Institute of Technology (BIT), Ranchi, India, in 1968, 1970, and 1979, respectively.

He joined BIT as Senior Research Fellow in 1970 and became Associate Professor in 1980. From 1983 to 1988 , he was with the University of Dar es Salaam (UDSM), Tanzania, where he became Professor in Telecommunications at the Department of Electrical Engineering in 1986. Since February 1988, he has been with the Telecommunications and Traffic Control Systems Group, Delft University of Technology, The Netherlands, where he is actively involved in the area of mobile, indoor and personal radio communications. While he was with BIT, he supervised many research projects in the area of microwave and plasma engineering. At UDSM he was responsible for the collaborative project "Satellite Communications for Rural Zones" with Eindhoven University of Technology, The Netherlands. He has published over 150 technical papers. His current research interest lies in packet communications, adaptive equalizers, spread-spectrum CDMA systems, and multimedia communications.

Dr. Prasad has served as a member of advisory and program commiteees of several IEEE international conferences. He has also presented tutorials on mobile and indoor radio communications at various universities, technical institutions, and IEEE conferences. He is also a member of a working group of European cooperation in the field of scientific and technical research (COST-231) project dealing with "Evolution of Land Mobile Radio (including personal) Communications" as an expert for the Netherlands. He is listed in the U.S. Who's Who in the World. He was Organizer and Interim Chairman of IEEE Vehicular Technology/Communications Society Joint Chapter, Benelux Section. Now he is the elected chairman of the joint chapter. He is also founder of the IEEE Symposium on Communications and Vehicular Technology (SCVT) in the Benelux and he was the Symposium Chairman of SCVT'93. He is one of the Editors-in-Chief of a new journal on "Wireless Personal Communications" and also a member of the editorial board of other international journals including IEEE COMMUNiCATIONS MAGAZINE. He is the Technical Program Chairman of PIMRC'94 International Symposium, held in The Hague, The Netherlands, September 19-23, 1994, and also of the Third Communication Theory Mini-Conference in conjunction with GLOBECOM'94, held in San Francisco, CA, November 27-30, 1994. He is a Fellow of IEE, a Fellow of the Institution of Electronics and Telecommunication Engineers, and a Member of the New York Academy of Sciences and NERG (The Netherlands Electronics and Radio Society). 